\title{
Effect of corn flour and storage period on sensory and physiochemical properties of chicken meatball
}

\author{
MA Al-Mamun, M Khan and MA Hashem*
}

Affiliation: Department of Animal Science, Bangladesh Agricultural University, Mymensingh-2202, Bangladesh

\begin{abstract}
The present study was undertaken to evaluate the effect of different levels of corn flour on the qualit characteristics of chicken meatball. The meatballs were formulated having 0, 05, 10 and $15 \%$ of cor flour. After formulation, samples were preserved at $-20^{\circ} \mathrm{C}$ for 60 days and analyzed the data at $0,15,3$ and $60^{\text {th }}$ day, respectively. The products were analyzed for various sensory, proximate, biochemical an physicochemical attributes. The sensory (color, flavor, tenderness, juiciness and overall acceptability. proximate composition- dry matter (DM), ether extract (EE), crude protein (CP) and ash, biochemici parameters- free fatty acid (FFA), peroxide value (PV) and thiobarbituric acid value (TBARS) an physicochemical (raw $\mathrm{pH}$, cooked $\mathrm{pH}$ and cooking loss) were analyzed. Data were analyzed in a $4 \mathrm{x}$ factorial experiment in completely randomized design with replicated three times per cell. Results showe that corn flour inclusion in meatballs have no effect on sensory parameters, but the highest value for a sensory parameters was found at $15^{\text {th }}$ day storage period, $(p<0.05)$. After $15^{\text {th }}$ day, with the increasing $c$ storage period the value for all sensory parameters were decreased $(p<0.05)$. The proximate component value were differ with treatment and day interval group $(p<0.05)$. The highest value of raw $\mathrm{pH}$ and lowes value of cooking loss were found in $5 \%$ corn flour group $(p<0.05)$. The highest value of cooked $\mathrm{pH}$ an lowest value of cooking loss were found at $15^{\text {th }}$ day storage period. There were no effect of treatment $c$ day interval on FFA and TBARS. Highest value of PV were found in $0 \%$ cornflower group $(p<0.05)$. It ma be concluded that $5 \%$ corn flour and 15 days storage period is suitable for chicken meat ball.
\end{abstract}

Key words: chicken, meatball, corn flour, sensory parameters

Bangladesh Animal Husbandry Association. All rights reserved.

Bang. J. Anim. Sci. 2017. 46 (3): 164-171

\section{Introduction}

Various types of meat products are prepared from meat in worldwide. Among the different meat products meatball is one of the tasty and popular ready foods. To gain economic benefits, the substitution of beef in meatball with meat of lower price such as chicken takes place frequently; a major portion of available meat usually comes from commercial broilers (Nahar et al., 2007). Unlike olden days where man used to have his food lavishly and slowly, the present trend changed the habits of foods, which are simple and easy to digest. Hence, the existence of these foods fulfilled all the needs of modern human being. Canned foods, convenience foods, fast foods, frozen foods, instant products, dried foods, preserved foods, etc. all comes under ready-to-eat foods. Non meat ingredients play a significant role in the modification of functional properties such as emulsification, and water and fat binding capacity, which may impact the textural properties. The liquid loss that occurs during cooking of processed meats may be reduced by the use of appropriate additives, which include carbohydrates, proteins, salt and phosphates by Hsu and Yu (1999).
Most Bangladeshi meatballs are manufactured by adding starch to provide desirable texture and cut down manufacturing costs. The essential ingredients that determine the quality of meatballs are flour (starch), water and fat or oil. Although meatballs are a popular food among consumers, there is a rising concern about the nutritive value of meatballs. The uses of rice bran profoundly affect the sensory and physicochemical properties of emulsified pork meatballs (Huang et al., 2005). Health benefits of wheat-based food products such oat bran addition in meatball decreases the total concentration of fat was found by Ylmaz and Dagloglu (2003). According to Jamaly et al., (2017) addition of $5 \%$ wheat flour can be used for the better performance of beef meatball in terms of sensory appraisal, physiochemical properties and microbial qualities. Freezing is the only known method by which meatball can be preserved in a condition similar to their normal state. Duration of storage period at freezer affects on the meatball quality. Based on the above discussion the present study was undertaken to identify the acceptable corn flour level and preferable storage duration on sensory and physiochemical parameters. 


\section{Al-Mamun et al. (2017) Bang. J. Anim. Sci. 46 (3):164-171}

\section{Materials and Methods}

\section{Place, duration and sample collection}

The experiment was conducted at the Animal Science Laboratory under the department of Animal Science, Bangladesh Agricultural University (BAU), Mymensingh, Bangladesh, during January to June 2015. Chicken meats of $2.5 \mathrm{~kg}$ freshly slaughtered chicken were collected from K.R. Market, Bangladesh Agricultural University (BAU), Mymensingh at 10.00 a.m. The meat samples were transferred to the Animal Science Laboratory immediately.

\section{Experimental design}

The present study was undertaken to evaluate the effect of different levels of corn flour on the quality characteristics of chicken meatball. The meatballs were formulated as four treatment groups having $\mathrm{T} 1-0 \%$ (control group), $\mathrm{T}_{2}-5 \%, \mathrm{~T}_{3}-10 \%, \mathrm{~T}_{4}-15 \%$ corn flour. After formulation, samples were preserved at $-20^{\circ} \mathrm{C}$ for 60 days and analyzed the data at $0,15,30$ and $60^{\text {th }}$ day, respectively. The sensory (color, flavor, tenderness, juiciness and overall acceptability), proximate components (dry matter, crude protein, ether extract, ash), physicochemical properties ( $\mathrm{pH}$ and cooking loss) and biochemical properties (free fatty acid, peroxide and thiobarbituric acid value) were analyzed for all treatment groups and day intervals.

\section{Preparation of meatball}

At first the chicken meats were properly cleaned with fresh water and the fat were trimmed off with sharp knife. Then the meat were grinded properly and mixed with garlic pest, onion pest, ginger pest, dry meat spices mix, egg yolk, cookies crumbs, ice flakes, refined vegetable oil. After that the mixed sample was aliquot into 4 parts. Then $0 \%, 05 \%$, $10 \%$ and $15 \%$ corn flower were mixed with four parts of mixed meat sample separately. Then meatballs of proper shape $(20-25 \mathrm{~g})$ were prepared separately from each group. Prepared meatballs were then boiled in hot water $\left(100^{\circ} \mathrm{C}\right)$ for $2-3$ minutes, water were removed from the meatball properly and fired in hot oil $\left(100^{\circ} \mathrm{C}\right)$ until reddish brown color obtained (3-4 min.). After frying the meatballs they were packaged in polyethylene bags as per treatment group and labeled carefully. Then they were kept at $-20^{\circ} \mathrm{C}$ for the pending analysis.

\section{Sensory evaluation}

Each meatball sample was evaluated by a trained 6-member panel. The sensory questionnaires measured intensity on a 5-point balanced semantic scale (weak to strong) for the attributes of color, smell, tenderness, juiciness, and overall acceptability. Sensory scores were 5 for excellent, 4 for very good, 3 for good, 2 for fair and 1 for poor (Rahman et al., 2012). Panelists were selected among department staff and students and trained according to the American Meat Science Association guidelines (AMSA, 1995).

\section{Proximate Composition}

Proximate composition was carried out according to the methods (AOAC, 2016). All determinations were done in triplicate and the mean values were reported.

\section{Biochemical analysis}

There were three types of biochemical analysis. These are Free Fatty Acid (FFA), Peroxide Value (POV) and Thiobarbituric Acid value (TBARS). FFA value was determined according to Rukunudin et al. (1998). POV was determined according to Sallam et al. (2004). Lipid oxidation was assessed in triplicate using the 2-thiobarbituric acid (TBA) method described by Schmedes and Holmer et al. (1989).

\section{Physiochemical analysis}

The $\mathrm{pH}$ value of raw and cooked meatball was measured using $\mathrm{pH}$ meter from raw and cooked meatball homogenate respectively. The homogenate was prepared by blending $5 \mathrm{~g}$ of meatball with $10 \mathrm{ml}$ distilled water. To determine the cooking loss firstly weighted meatballs boiled at water bath to $100^{\circ} \mathrm{C}$. After completed boiling samples were removed from the water bath and egg albumin, biscuit crumbs were mixed with all types of chicken meatballs. Again all samples were cooked with refined soybean oil to $100^{\circ} \mathrm{C}$. After completed the frying of meatballs these are properly removed and were kept in cooled condition for to get at room temperature. Then the cooking loss was measured on per cent basis.

\section{Statistical analysis}

Data were statistically analyzed using SAS statistical discovery software, NC, USA. DMRT test was used to determine the significance of differences among treatments means. Means were considered significantly different for $p<0.05$. Data presented are shown as means \pm SD. 
Table 1. Effect of corn flour on sensory parameters of chicken meatball

\begin{tabular}{|c|c|c|c|c|c|c|c|c|c|}
\hline \multirow{2}{*}{$\begin{array}{l}\text { Parame } \\
\text { ters }\end{array}$} & \multirow{2}{*}{ DI } & \multicolumn{4}{|c|}{ Treatments } & \multirow[t]{2}{*}{ Mean } & \multicolumn{3}{|c|}{ Level of significance } \\
\hline & & $\mathbf{T}_{\mathbf{1}}$ & $\mathbf{T}_{2}$ & $T_{3}$ & $\mathbf{T}_{4}$ & & Treat. & DI & $\mathbf{T} * \mathbf{D I}$ \\
\hline \multirow{5}{*}{ Color } & 0 & $4.33 \pm 0.33$ & $4.66 \pm 0.33$ & $4.66 \pm 0.33$ & $4.66 \pm 0.33$ & $4.58^{a} \pm 0.33$ & \multirow{5}{*}{0.3414} & \multirow{5}{*}{0.006} & \multirow{5}{*}{0.8421} \\
\hline & 15 & $4.33 \pm 0.33$ & $4.66 \pm 0.33$ & $4.66 \pm 0.33$ & $5.00 \pm 0.00$ & $4.66^{a} \pm 0.24$ & & & \\
\hline & 30 & $4.33 \pm 0.33$ & $3.66 \pm 0.33$ & $4.00 \pm 0.57$ & $4.33 \pm 0.33$ & $3.83^{b} \pm 0.39$ & & & \\
\hline & 60 & $4.66 \pm 0.33$ & $4.33 \pm 0.33$ & $4.66 \pm 0.33$ & $4.33 \pm 0.33$ & $4.50^{\mathrm{a}} \pm 0.33$ & & & \\
\hline & Mean & $4.16^{\mathrm{a}} \pm 0.33$ & $4.33^{\mathrm{a}} \pm 0.33$ & $4.50^{\mathrm{a}} \pm 0.39$ & $4.58^{\mathrm{a}} \pm 0.24$ & & & & \\
\hline \multirow{5}{*}{ Odor } & 0 & $4.33 \pm 0.33$ & $4.66 \pm 0.33$ & $4.66 \pm 0.33$ & $5.00 \pm 0.00$ & $4.66^{\mathrm{a}} \pm 0.24$ & \multirow{5}{*}{0.644} & \multirow{5}{*}{0.0031} & \multirow{5}{*}{0.9890} \\
\hline & 15 & $4.33 \pm 0.33$ & $4.66 \pm 0.33$ & $4.66 \pm 0.33$ & $5.00 \pm 0.00$ & $4.66^{\mathrm{a}} \pm 0.24$ & & & \\
\hline & 30 & $3.33 \pm 0.33$ & $3.66 \pm 0.33$ & $4.00 \pm 0.57$ & $4.33 \pm 0.33$ & $3.83^{b} \pm 0.39$ & & & \\
\hline & 60 & $4.33 \pm 0.33$ & $4.66 \pm 0.33$ & $4.33 \pm 0.33$ & $4.66 \pm 0.33$ & $4.50^{\mathrm{a}} \pm 0.33$ & & & \\
\hline & Mean & $4.08^{b} \pm 0.33$ & $4.41^{\mathrm{ab}} \pm 0.33$ & $4.41^{\mathrm{ab}} \pm 0.39$ & $4.75^{\mathrm{a}} \pm 0.16$ & & & & \\
\hline \multirow{5}{*}{$\begin{array}{l}\text { Tendern } \\
\text { ess }\end{array}$} & 0 & $4.66 \pm 0.33$ & $4.66 \pm 0.33$ & $5.00 \pm 0.00$ & $4.66 \pm 0.33$ & $4.75^{\mathrm{a}} \pm 0.24$ & \multirow{5}{*}{0.1779} & \multirow{5}{*}{$0.000 \Xi$} & \multirow{5}{*}{0.1194} \\
\hline & 15 & $4.66 \pm 0.33$ & $4.66 \pm 0.33$ & $4.66 \pm 0.33$ & $5.00 \pm 0.00$ & $4.75^{a} \pm 0.24$ & & & \\
\hline & 30 & $3.33 \pm 0.33$ & $4.00 \pm 0.00$ & $4.33 \pm 0.33$ & $4.66 \pm 0.33$ & $4.08^{b} \pm 0.25$ & & & \\
\hline & 60 & $4.33 \pm 0.33$ & $3.33 \pm 0.33$ & $4.33 \pm 0.33$ & $3.66 \pm 0.33$ & $3.91^{\mathrm{b}} \pm 0.33$ & & & \\
\hline & Mean & $4.25^{\mathrm{a}} \pm 0.33$ & $4.16^{\mathrm{a}} \pm 0.24$ & $4.58^{\mathrm{a}} \pm 0.24$ & $4.50^{\mathrm{a}} \pm 0.16$ & & & & \\
\hline \multirow{5}{*}{ Juicines } & 0 & $4.66 \pm 0.33$ & $4.66 \pm 0.33$ & $4.33 \pm 0.33$ & $5.00 \pm 0.00$ & $4.66^{a} \pm 0.24$ & \multirow{5}{*}{0.4574} & \multirow{5}{*}{0.0169} & \multirow{5}{*}{0.955} \\
\hline & 15 & $4.66 \pm 0.33$ & $4.66 \pm 0.33$ & $4.66 \pm 0.33$ & $5.00 \pm 0.00$ & $4.75^{a} \pm 0.24$ & & & \\
\hline & 30 & $3.66 \pm 0.33$ & $3.66 \pm 0.33$ & $4.00 \pm 0.57$ & $4.33 \pm 0.33$ & $3.19^{b} \pm 0.39$ & & & \\
\hline & 60 & $4.66 \pm 0.33$ & $4.66 \pm 0.33$ & $4.00 \pm 0.57$ & $4.33 \pm 0.33$ & $4.33^{\mathrm{ab}} \pm 0.39$ & & & \\
\hline & Mean & $4.33^{\mathrm{a}} \pm 0.33$ & $4.33^{a} \pm 0.33$ & $4.25^{\mathrm{a}} \pm 0.45$ & $4.66^{a} \pm 0.16$ & & & & \\
\hline \multirow{5}{*}{$\begin{array}{l}\text { Overall } \\
\text { accepta } \\
\text { bility }\end{array}$} & 0 & $4.33 \pm 0.33$ & $4.66 \pm 0.33$ & $4.66 \pm 0.33$ & $4.66 \pm 0.33$ & $4.58^{a} \pm 0.33$ & \multirow{5}{*}{0.2574} & \multirow{5}{*}{0.0067} & \multirow{5}{*}{0.5884} \\
\hline & 15 & $4.66 \pm 0.33$ & $4.66 \pm 0.33$ & $4.66 \pm 0.33$ & $5.00 \pm 0.00$ & $4.66^{a} \pm 0.24$ & & & \\
\hline & 30 & $3.33 \pm 0.33$ & $3.66 \pm 0.33$ & $4.00 \pm 0.57$ & $4.33 \pm 0.33$ & $3.83^{b} \pm 0.39$ & & & \\
\hline & 60 & $4.66 \pm 0.33$ & $3.66 \pm 0.33$ & $4.33 \pm 0.57$ & $4.33 \pm 0.33$ & $4.25^{\mathrm{ab}} \pm 0.39$ & & & \\
\hline & Mean & $4.16^{\mathrm{a}} \pm 0.33$ & $4.16^{a} \pm 0.33$ & $4.41^{a} \pm 0.45$ & $4.58^{a} \pm 0.24$ & & & & \\
\hline
\end{tabular}

Column mean value having different superscript varies significantly at values $p<0.05$. Again, mean values having same superscript in each row did not differ significantly at $\mathrm{p}>0.05$. T1, Control group; T2, 05\% corn flour group; T3, 10\% corn flour group; T4, 5\% corn flour group; DI, Day Intervals; Treat, Treatment; T*DI, Interaction of Treatment and Day Interval 
Al-Mamun et al. (2017) Bang. J. Anim. Sci. 46 (3):164-171

Table 2. Effect of corn flour on chemical composition of chicken meatball

\begin{tabular}{|c|c|c|c|c|c|c|c|c|c|}
\hline \multirow{2}{*}{$\begin{array}{l}\text { Paramei } \\
\text { ers }\end{array}$} & \multirow[t]{2}{*}{ DI } & \multicolumn{4}{|c|}{ Treatments } & \multirow[t]{2}{*}{ Mean } & \multicolumn{3}{|c|}{ Level of significance } \\
\hline & & $\mathbf{T}_{1}$ & $\mathbf{T}_{\mathbf{2}}$ & $\mathbf{T}_{\mathbf{3}}$ & $\mathbf{T}_{4}$ & & Treat. & DI & $\mathbf{T} * \mathbf{D I}$ \\
\hline \multirow{5}{*}{ DM (\%) } & 0 & $53.61 \pm 0.09$ & $47.21 \pm 0.14$ & $45.61 \pm 0.0$ & $54.0 \pm 0.09$ & $50.11^{\mathrm{a}} \pm 0.10$ & \multirow{10}{*}{$<0.000$} & \multirow{5}{*}{$<0.0001$} & \multirow{5}{*}{$<0.0001$} \\
\hline & 15 & $50.46 \pm 0.49$ & $47.93 \pm 0.12$ & $54.08 \pm 0.5$ & $46.84 \pm 0.06$ & $49.83^{a} \pm 0.29$ & & & \\
\hline & 30 & $51.08 \pm 0.61$ & $48.02 \pm 0.1 \varepsilon$ & $53.65 \pm 0.01$ & $47.15 \pm 0.43$ & $49.97^{a} \pm 0.30$ & & & \\
\hline & 60 & $51.53 \pm 0.51$ & $49.52 \pm 0.57$ & $46.46 \pm 0.31$ & $47.18 \pm 0.30$ & $48.67^{b} \pm 0.43$ & & & \\
\hline & Mean & $\begin{array}{l}\quad 51.67^{\mathrm{a}} \pm \\
0.42\end{array}$ & $48.17^{d} \pm 0.2^{\mathrm{s}}$ & $\begin{array}{l}49.95^{b} \pm \\
0.23\end{array}$ & $48.97^{c} \pm 0.22$ & & & & \\
\hline \multirow{5}{*}{$\mathrm{CP}(\%)$} & 0 & $21.37 \pm 0.29$ & $20.84 \pm 0.17$ & $20.15 \pm 0.1$ & $20.23 \pm 0.09$ & $20.65^{\mathrm{a}} \pm 0.16$ & & \multirow{5}{*}{0.1656} & \multirow{5}{*}{0.8834} \\
\hline & 15 & $21.05 \pm 0.06$ & $20.18 \pm 0.27$ & $19.75 \pm 0.4 i$ & $20.13 \pm 0.38$ & $20.28^{\mathrm{a}} \pm 0.29$ & & & \\
\hline & 30 & $21.05 \pm 0.45$ & $20.24 \pm 0.12$ & $19.69 \pm 0.1 i$ & $20.07 \pm 0.04$ & $20.26^{\mathrm{a}} \pm 0.19$ & & & \\
\hline & 60 & $21.31 \pm 0.06$ & $20.60 \pm 0.11$ & $19.66 \pm 0.3$ & $19.65 \pm 0.47$ & $20.11^{\mathrm{a}} \pm 0.24$ & & & \\
\hline & Mean & $21.19^{a} \pm 0.21$ & $20.47^{b} \pm 0.1 \epsilon$ & $\begin{array}{l}19.81^{\mathrm{c}} \pm \\
0.27\end{array}$ & $20.02^{c} \pm 0.24$ & & & & \\
\hline \multirow{5}{*}{$\mathrm{EE}(\%)$} & 0 & $7.85 \pm 0.26$ & $8.53 \pm 0.33$ & $8.89 \pm 0.13$ & $8.67 \pm 0.09$ & $8.49^{a} \pm 0.20$ & \multirow{5}{*}{0.0003} & \multirow{5}{*}{0.0154} & \multirow{5}{*}{0.0106} \\
\hline & 15 & $8.33 \pm 0.18$ & $7.70 \pm 0.12$ & $8.62 \pm 0.18$ & $7.49 \pm 0.25$ & $8.03^{b} \pm 0.18$ & & & \\
\hline & 30 & $8.23 \pm 0.02$ & $7.74 \pm 0.06$ & $8.49 \pm 0.06$ & $7.52 \pm 0.06$ & $7.99^{b} \pm 0.05$ & & & \\
\hline & 60 & $7.97 \pm 0.38$ & $8.56 \pm 0.36$ & $8.41 \pm 0.30$ & $7.63 \pm 0.19$ & $8.14^{b} \pm 0.30$ & & & \\
\hline & Mean & $8.09^{b} \pm 0.21$ & $8.13^{b} \pm 0.21$ & $8.60^{a} \pm 0.1 \epsilon$ & $7.83^{b} \pm 0.14$ & & & & \\
\hline \multirow{5}{*}{ Ash (\%) } & 0 & $1.24 \pm 0.07$ & $1.27 \pm 0.06$ & $1.24 \pm O C$ & $1.31 \pm 00$ & $1.26^{\mathrm{ab}} \pm 0.03$ & \multirow{5}{*}{0.0194} & \multirow{5}{*}{0.0016} & \multirow{5}{*}{0.3993} \\
\hline & 15 & $1.20 \pm 0.04$ & $1.13 \pm 0.03$ & $\begin{array}{l}1.19 \pm \\
0.03\end{array}$ & $1.25 \pm 0.02$ & $1.16^{c} \pm 0.03$ & & & \\
\hline & 30 & $1.21 \pm 0.04$ & $1.21 \pm 0.05$ & $1.21 \pm 0.08$ & $1.32 \pm 0.05$ & $1.21^{\mathrm{bc}} \pm 0.05$ & & & \\
\hline & 60 & $1.37 \pm 0.01$ & $1.34 \pm 0.03$ & $1.25 \pm 0.10$ & $1.37 \pm 0.07$ & $1.33^{\mathrm{a}} \pm 0.05$ & & & \\
\hline & Mean & $1.25^{\mathrm{ab}} \pm 0.04$ & $1.18^{b} \pm 0.04$ & $1.22^{b} \pm 0.0 \mathrm{~s}$ & $1.31^{\mathrm{a}} \pm 0.03$ & & & & \\
\hline
\end{tabular}

Column mean value having different superscript varies significantly at values $p<0.05$. Again, mean values having same superscript in each row did not differ significantly at $\mathrm{p}>0.05$. T1, Control group; T2, 05\% corn flour group; T3, 10\% corn flour group; T4, 5\% corn flour group; DI, Day Intervals; Treat, Treatment; T*DI, Interaction of Treatment and Day Interval 


\section{Corn flour on chicken meatball}

\section{Results and Discussion}

\section{Sensory Evaluation}

The observation of sensory evaluation of different treatments with day intervals is shown in Table 1. The range of color score at different treatment was 4.16 to 4.58 , and 4.58 to 3.83 for days intervals. The most preferable color was observed on $15^{\text {th }}$ day storage period. The decreased color scores during storage resulted from the denaturation of proteins, particularly the myofibrillar protein that affects gel formation Descalzo and Sancho (2008). The range of odor score among four treatments was 4.08 to 4.75 , and 3.83 to 4.66 for days intervals. There was significant difference $(p<0.05)$ due to days interval of odor of meatballs. The values of odor deteriorated with increased storage period. Odor is one of the major causes of quality deterioration (Raghavan et al., 2007) because it can negatively affect sensory attributes such as color, texture as well as the nutritional quality of the product mentioned by Nunez and Boleman (2008). The range of tenderness score at different treatments was 4.16 to 4.58 , and 3.91 to 4.75 for days interval. The most preferable tenderness was observed on 0 and $15^{\text {th }}$ day $(p<0.05)$, and after that the preference of tenderness were decrease with increasing the storage period. The result of this experiment is agreed to the results of Lui et al. (2010). The range of juiciness score at different treatments was 4.25 to 4.66 , and 3.19 to 4.75 for days interval. The range of overall acceptability score at different treatments was 4.16 to 4.58 , and 3.83 to 4.58 for days interval. The most preferable overall acceptability was observed on $15^{\text {th }}$ day $(p<0.05)$

\section{Proximate components}

The values of proximate components are shown in Table 2. Similar results were reported for Indonesian traditional meatballs with a DM content ranged from 56.17 to $60.32 \%$ mentioned by Purnomo and Rahardiyan (2008). The range of CP content at different treatments was 21.19 to $19.81 \%$, and 20.11 to $20.65 \%$ for days intervals. The highest amount of $\mathrm{CP}$ content indicates this product is most preferable to consumer. Data show that the lowest amount of CP content was $20.11 \%$ after 60 days of storage period. Traditional koefte meatballs showed higher protein content $(25.51 \%)$ reported by Ulu (2004). The range of different days of intervals of EE content was 7.99 to $8.49 \%$. Lowfat traditional Turkey beef meatballs had similar fat content ranged from 7.9 to $8.8 \%$ reported by Serdaroglu et al. (2005). Verma et al. (2009) reported significant $(p<0.05)$ decrease in low fat chicken nuggets incorporated with chickpea hull flour. The range of ash content at different treatments was to 1.18 to $1.31 \%$, and 1.16 to $1.33 \%$ for days intervals. Similar results were also reported by Serdaroglu et al (2005) on the ash content of beef meatballs, which ranged from 2.6 to $2.8 \%$.

\section{Physicochemical properties}

The values of raw $\mathrm{pH}$, cooked $\mathrm{pH}$ and cooking loss are shown in the Table 3. The range of raw $\mathrm{pH}$ at different treatments was 5.55 to 5.75 , and 5.48 to 5.82 for days intervals. Among these four treatments most preferable raw $\mathrm{pH}$ was observed from $5 \%$ corn flour group. The highest amount of raw $\mathrm{pH}$ indicates this product is most preferable for consumer's health. Bacteria and mold have a tendency to increase with increasing storage time, and they secrete components that affect the increasing raw $\mathrm{pH}$. The range of cooked $\mathrm{pH}$ at different treatments was 5.99 to 6.03 , and 5.97 to 6.05 due to days intervals. These results are similar to those of Sallam et al. (2004), who reported that storage time had a significant $(p<0.05)$ effect on $\mathrm{pH}$ values. Similar results have also been found in the study of antioxidant treatments during storage time using a mixture of $\mathrm{BHA}$ and $\mathrm{BHT}$ in precooked pork patties by Biswas et al. (2004). The range cooking loss at different treatments was 26.46 to $27.54 \%$, and 26.11 to $27.35 \%$ for days intervals. Among these four treatments most preferable cooking loss was observed from $5 \%$ corn flour group. The lowest amount of cooking loss indicates this product is most preferable to consumers' choices. Major components of cooking losses are thawing, dripping and evaporation. Thawing loss refers to the loss of fluid in meatballs resulting from the formation of exudates following freezing and thawing by Jama, et al. (2008), Muchenje, et al. (2009). Dripping is the loss of fluid from meatballs and water evaporation from the shrinkage of muscle proteins by $\mathrm{Yu}$, et al. (2005).

\section{Biochemical properties}

The value of biochemical components are shown in Table 4. The range of FFA value at different treatments was 0.32 to $0.33 \%$ and 0.32 to $0.34 \%$ for days intervals. Similar results also found in the study of Jamaly et al. (2017). During storage, the POV increased in all treatments. 
Al-Mamun et al. (2017) Bang. J. Anim. Sci. 46 (3):164-171

Table 3. Effect of corn flour as a source of fiber of chicken meatball

\begin{tabular}{|c|c|c|c|c|c|c|c|c|c|}
\hline \multicolumn{3}{|c|}{ DI } & \multicolumn{3}{|c|}{ Treatments } & \multirow[t]{2}{*}{ Mean } & \multicolumn{3}{|c|}{ Level of significance } \\
\hline & & $\mathbf{T}_{1}$ & $\mathbf{T}_{2}$ & $T_{3}$ & $\overline{T_{4}}$ & & Treat. & DI & $\mathbf{T} * \mathbf{D I}$ \\
\hline \multirow{5}{*}{ Raw pH } & 0 & $5.87 \pm 0.03$ & $5.86 \pm 0.04$ & $5.81 \pm 0.06$ & $5.76 \pm 0.10$ & $5.82^{\mathrm{a}} \pm 0.05$ & \multirow{5}{*}{0.0595} & \multirow{5}{*}{0.0017} & \multirow{5}{*}{0.0566} \\
\hline & 15 & $5.52 \pm 0.07$ & $6.00 \pm 0.06$ & $5.49 \pm 0.06$ & $5.69 \pm 0.06$ & $5.67^{\mathrm{ab}} \pm 0.0 \mathrm{t}$ & & & \\
\hline & 30 & $5.62 \pm 0.13$ & $5.81 \pm 0.17$ & $5.45 \pm 0.08$ & $5.45 \pm 0.06$ & $5.58^{\mathrm{bc}} \pm 0.1$ & & & \\
\hline & 60 & $5.76 \pm 0.17$ & $5.33 \pm 0.18$ & $5.47 \pm 0.22$ & $5.35 \pm 0.07$ & $5.48^{c} \pm 0.1 \epsilon$ & & & \\
\hline & Mean & $5.69^{\mathrm{ab}} \pm 0.10$ & $5.75^{\mathrm{a}} \pm 0.11$ & $5.55^{b} \pm 0.10$ & $5.56^{\mathrm{b}} \pm 0.07$ & & & & \\
\hline \multirow{5}{*}{ Cooked pH } & 0 & $5.97 \pm 0.00$ & $5.97 \pm 0.03$ & $5.96 \pm 0.03$ & $5.99 \pm 0.05$ & $5.97^{b} \pm 0.02$ & \multirow{5}{*}{0.42} & \multirow{5}{*}{0.01} & \multirow{5}{*}{0.588} \\
\hline & 15 & $6.03 \pm 0.01$ & $6.09 \pm 0.00$ & $6.03 \pm 0.01$ & $6.05 \pm 0.03$ & $6.05^{a} \pm 0.01$ & & & \\
\hline & 30 & $5.98 \pm 0.01$ & $6.07 \pm 0.06$ & $5.97 \pm 0.01$ & $6.00 \pm 0.02$ & $6.00^{b} \pm 0.02$ & & & \\
\hline & 60 & $6.01 \pm 0.04$ & $5.98 \pm 0.01$ & $6.02 \pm 0.02$ & $5.99 \pm 0.02$ & $6.00^{b} \pm 0.02$ & & & \\
\hline & Mean & $5.99^{\mathrm{a}} \pm 0.01$ & $6.03^{a} \pm 0.0 z$ & $5.99^{\mathrm{a}} \pm 0.01$ & $6.01^{\mathrm{a}} \pm 0.03$ & & & & \\
\hline \multirow{5}{*}{$\begin{array}{c}\text { Cooking loss } \\
(\%)\end{array}$} & 0 & $27.54 \pm 0.15$ & $26.48 \pm 0.0 ؟$ & $28.34 \pm 0.13$ & $27.06 \pm 0.1<$ & $27.35^{\mathrm{a}} \pm 0.1$ & \multirow{5}{*}{$<.0001$} & \multirow{5}{*}{$<.0001$} & \multirow{5}{*}{0.0039} \\
\hline & 15 & $25.74 \pm 0.16$ & $25.54 \pm 0.1\{$ & $27.03 \pm 0.07$ & $26.12 \pm 0.0\{$ & $26.11^{c} \pm 0.1$ & & & \\
\hline & 30 & $26.66 \pm 0.47$ & $26.45 \pm 0.0 t$ & $27.49 \pm 0.15$ & $26.53 \pm 0.0$ & $26.78^{\mathrm{b}} \pm 0.1$ & & & \\
\hline & 60 & $27.40 \pm 0.28$ & $27.37 \pm 0.3 !$ & $27.32 \pm 0.05$ & $27.20 \pm 0.1:$ & $27.32^{\mathrm{a}} \pm 0.2$ & & & \\
\hline & Mean & $26.83^{b} \pm 0.26$ & $26.46^{\mathrm{C}} \pm 0.1$ & $27.54^{\mathrm{a}} \pm 0.1 \mathrm{c}$ & $\begin{array}{c}26.73^{\mathrm{bc}} \pm \\
0.09\end{array}$ & & & & \\
\hline
\end{tabular}

Column mean value having different superscript varies significantly at values $p<0.05$. Again, mean values having same superscript in each row did not differ significantly at $\mathrm{p}>0.05$. T1, Control group; T2, 05\% corn flour group; T3, 10\% corn flour group; T4, 5\% corn flour group; DI, Day Intervals; Treat, Treatment; T*DI, Interaction of Treatment and Day Interval

Table 4. Effect of corn flour on biochemical properties of chicken meatball

\begin{tabular}{|c|c|c|c|c|c|c|c|c|c|}
\hline \multirow[t]{2}{*}{ Parameters } & \multirow[t]{2}{*}{ DI } & \multicolumn{4}{|c|}{ Treatments } & \multirow[t]{2}{*}{ Mean } & \multicolumn{3}{|c|}{ Level of significance } \\
\hline & & $\mathbf{T}_{1}$ & $\mathbf{T}_{\mathbf{2}}$ & $\mathbf{T}_{\mathbf{3}}$ & $\mathbf{T}_{4}$ & & Treat. & DI & $\mathbf{T} * \mathbf{D I}$ \\
\hline \multirow{5}{*}{ FFA (\%) } & 0 & $0.33 \pm 0.01$ & $0.33 \pm 0.00$ & $0.31 \pm 0.00$ & $0.34 \pm 0.01$ & $0.33^{a} \pm 0.00$ & \multirow{5}{*}{0.711} & \multirow{5}{*}{0.165} & \multirow{5}{*}{0.455} \\
\hline & 15 & $0.33 \pm 0.00$ & $0.32 \pm 0.00$ & $0.33 \pm 0.00$ & $0.32 \pm 0.01$ & $0.32^{\mathrm{a}} \pm 0.00$ & & & \\
\hline & 30 & $0.34 \pm 0.00$ & $0.31 \pm 0.00$ & $0.35 \pm 0.01$ & $0.34 \pm 0.01$ & $0.33^{a} \pm 0.00$ & & & \\
\hline & 60 & $0.34 \pm 0.00$ & $0.33 \pm 0.01$ & $0.35 \pm 0.01$ & $0.35 \pm 0.00$ & $0.34^{a} \pm 0.00$ & & & \\
\hline & Mean & $0.33^{a} \pm 0.0 c$ & $0.32^{\mathrm{a}} \pm 0.0 \mathrm{c}$ & $0.33^{a} \pm 0.0 C$ & $0.32^{a} \pm 0.00$ & & & & \\
\hline \multirow{5}{*}{ POV (meq/kg) } & 0 & $3.77 \pm 0.10$ & $3.73 \pm 0.13$ & $3.46 \pm 0.05$ & $2.99 \pm 0.07$ & $3.48^{a} \pm 0.08$ & \multirow{5}{*}{0.003} & \multirow{5}{*}{0.435} & \multirow{5}{*}{0.001} \\
\hline & 15 & $3.55 \pm 0.07$ & $3.72 \pm 0.06$ & $3.32 \pm 0.20$ & $3.63 \pm 0.08$ & $3.55^{a} \pm 0.10$ & & & \\
\hline & 30 & $3.61 \pm 0.09$ & $3.57 \pm 0.15$ & $2.99 \pm 0.07$ & $3.73 \pm 0.13$ & $3.47^{\mathrm{a}} \pm 0.11$ & & & \\
\hline & 60 & $3.75 \pm 0.07$ & $3.49 \pm 0.20$ & $3.58 \pm 0.06$ & $3.59 \pm 0.13$ & $3.60^{a} \pm 0.11$ & & & \\
\hline & Mean & $3.67^{a} \pm 0.0 !$ & $3.62^{\mathrm{a}} \pm 0.1 \Xi$ & $3.33^{b} \pm 0.0 \subseteq$ & $3.48^{\mathrm{ab}} \pm 0.10$ & & & & \\
\hline \multirow{5}{*}{$\begin{array}{c}\text { TBARS } \\
(\mathrm{mg}-\mathrm{MA} / \mathrm{kg})\end{array}$} & 0 & $0.09 \pm 0.01$ & $0.11 \pm 0.00$ & $0.11 \pm 0.00$ & $0.11 \pm 0.00$ & $0.10^{a} \pm 0.00$ & \multirow{5}{*}{0.317} & \multirow{5}{*}{0.40} & \multirow{5}{*}{0.433} \\
\hline & 15 & $0.10 \pm 0.00$ & $0.11 \pm 0.00$ & $0.11 \pm 0.00$ & $0.11 \pm 0.00$ & $0.10^{a} \pm 0.00$ & & & \\
\hline & 30 & $0.11 \pm 0.00$ & $0.11 \pm 0.00$ & $0.11 \pm 0.00$ & $0.11 \pm 0.00$ & $0.11^{a} \pm 0.00$ & & & \\
\hline & 60 & $0.11 \pm 0.00$ & $0.11 \pm 0.00$ & $0.11 \pm 0.00$ & $0.11 \pm 0.00$ & $0.11^{a} \pm 0.00$ & & & \\
\hline & Mean & $0.10^{\mathrm{a}} \pm 0.0 \mathrm{C}$ & $011^{a} \pm 0.00$ & $0.11^{\mathrm{a}} \pm 0.0 \mathrm{C}$ & $011^{a} \pm 0.00$ & & & & \\
\hline
\end{tabular}

Column mean value having different superscript varies significantly at values $p<0.05$. Again, mean values having same superscript in each row did not differ significantly at $\mathrm{p}>0.05$. T1, Control group; T2, 05\% corn flour group; T3, 10\% corn flour group; T4, 5\% corn flour group; DI, Day Intervals; Treat, Treatment; T*DI, Interaction of Treatment and Day Interval. 


\section{Corn flour on chicken meatball}

Novelli et al. (1998) also showed increasing POV with longer storage time in a sausage product. Rhee and Myers (2003) examined POV in plain goat meat loaf reported a similar trend in POV during storage. There was no effect of treatment on the TBARS. The range of different days of intervals of TBARS value was 0.10 to $0.11 \mathrm{mg}-\mathrm{MA} / \mathrm{kg}$. Das et al. (2012) observed that the Moringa oleiferia leaves extract was more effective to prevent increased TBARS number of precooked goat meat patties during storage. Similar results were observed for pork (Alvarez-Parrilla et al., 2012) and chicken products by Castro et al., (2011).

\section{Conclusion}

From this study it may be concluded there was no effect of corn flour on sensory parameters of chicken meatball. The highest value for all sensory parameters was found at $15^{\text {th }}$ day storage period. After $15^{\text {th }}$ day, with the increasing of storage period the value for all sensory parameters decreases gradually. The highest value of raw $\mathrm{pH}$ and lowest value of cooking loss were found in $5 \%$ corn flour group. It may be concluded that $5 \%$ corn flour and 15 days storage period is suitable for chicken meat ball.

\section{References}

Alvarez-Parrilla E, LA Rosa, de la, R Amarowicz and F Shahidi (2012). Protective effect of fresh and processed Jalapeño and Serrano peppers against food lipid and human LDL cholesterol oxidation. Food Chemistry 133(3): 827-834.

AMSA (1995). Research guidelines for cookery, sensory evaluation, and instrumental tenderness measurements of fresh meat. Chicago III. American Meat Science Association and Nutritional Live Stock and Meat Board.

AOAC (2016). Official Methods of Analysis. (20 th edition) Association of Official Analytical Chemists. Washington, DC, USA.

Biswas AK, RC Keshri and GS Bisht (2004). Effect of enrobing and antioxidants on quality characteristics of precooked pork patties under chilled and frozen storage conditions. Meat Science 66: 733-741.

Castro WF, LRB Mariutti and N Bragagnolo (2011). The effects of colorifico on lipid oxidation, colour and vitamin $\mathrm{E}$ in raw and grilled chicken patties during frozen storage. Food Chemistry 124(1): 126-131.
Das AK, V Rajkumar, AK Verma and D Swarup (2012). Moringa oleiferia leaves extract a natural antioxidant for retarding lipid peroxidation in cooked goat meat patties. International Journal of Food Science Technology 47: 585-591.

Descalzo AM and AM Sancho (2008). A review of natural antioxidants and their effects on oxidative status, odor and quality of fresh beef produced in Argentina. Meat Science 79: 423436.

Hsu SY and SH Yu (1999). Effects of phosphate, water, fat, and salt on qualities of low-fat emulsified meatball. Journal of food engineering 39: 123-130.

Huang SC, CY Shiau, TE Liu, CL Chu and DF Hwang (2005). Effects of rice bran on sensory and physico-chemical properties of emulsified pork meatballs. Meat Science 70(4) 613-619.

Huang SC, CY Shiau, TE Liu, CL Chu and DF Hwang (2005). Effects of rice bran on sensory and physico-chemical properties of emulsified pork meatballs. MeatScience 70(4) 613-619.

Jama N, V Muchenje, M Chimonyo, PE Strydom, K Dzama and JG Raats (2008). Cooking loss components of beef from Nguni, Bonsmara and Angus steers. African Journal of Agricultural Research 3 (6): 416-420.

Jamaly SI, MA Hashem, S Akther and MA Hossain (2017). Wheat flour as dietary fiber on fresh and preserved beef meatballs. Bangladesh Journal of Animal Science 46(1): 35-43.

Lui Z, Y Xiong and J Chen (2010). Protein oxidation enhances hydration but suppresses waterholding capacity in porcine longissimus muscle. Journal of Agricultural and Food Chemistry 58: 10697-10704.

Muchenje V, K Dzama, M Chimonyo, PE Strydom, A Hugo and JG Raats (2009). Some biochemical aspects pertaining to beef eating quality and consumer health: A review. Food Chemistry 112: 279-289.

Nahar K, S Ahmed, MM Monir and MS Rahman (2007). Comparative evaluation of egg quality characteristics of broiler parent stock and synthetic broiler. Bangladesh Journal of Animal Science 36(1 \& 2): 82-87.

Novelli E, E Zanardi, GP Ghiretti, G Campanini, G Dazzi, G Madarena and R Chizzolini (1998). 


\section{Al-Mamun et al. (2017) Bang. J. Anim. Sci. 46 (3):164-171}

Lipid and cholesterol oxidation in frozen stored pork, salameMilano and mortadella. Meat Science 48(1-2): 29-40.

Nunez G and S Boleman (2008). Microbial safety of meat in the European Union. Meat Science 78: 14-18.

Purnomo A, and D Rahardiyan (2008). Indonesian traditional meatball: Review article. Int. Food Res. J. 15: 101-108.

Raghavan SY, SS Richards, JB Eu, HC Lee, YJ Kim and KB Chin (2007). Evaluation of lipid oxidation and oxidative products as affected by pork meat cut, packaging, method and storage time during frogen storage. Journal of Food Science 72(2): C114-C119.

Rahman MR (2012). TBA test by an extractive method applied to pate. Meat science 42(1): 103-110.

Rhee KS and CE Myers (2003). Sensory properties and lipid oxidation in aerobically refrigerated cooked ground goat meat. Meat Science 66: 189-194.

Rukunudin IH, PJ White, CJ Bern and TB Bailey (1998). A modified method for determining free fatty acids from small soybean sample sizes. Journal of Ambion Oil Chemical Society 75: 563-568.

Sallam KI, M Ishioroshi and K Samejima (2004). Antioxidants and antimicrobial effects of garlic in chicken sausage. Lebensm. Wiss. Technology 37: 849-855.

Schmedes A and G Holmer (1989). A new thiobarbituric acid (TBA) method for determining free malondialdehyde (MDA) and hydroperoxides selectively as a measure of lipid peroxidation. Journal of Ambion Oil Chemical Society 66: 813-817.

Serdaroglu M, G YldzTurp and K Abrodimov (2005). Quality of low-fat meatballs containing Legume flours as extenders. Meat Science 70(1): 99-105.

Ulu H (2004). Effect of wheat flour, whey protein concentrate and soya protein isolate on oxidative process and textural properties of cooked meatballs. Food Chemistry 87: 523529.

Verma AR, M Vijayakumar, CS Mathela and CV Rao (2009). In vitro and in vivo antioxidant properties of different fractions of Moringa oleifera leaves. Food and Chemical Toxicology 47: 2196-2201.

Ylmaz I and O Dagloglu (2003). The effect of replacing fat with oat bran on fatty acid composition and physicochemical properties of meatballs. Meat Science 65(2) 819-823.

Yu LH, ES Lee, JY Jeong, HD Paik, JH Choi and CJ Kim (2005). Effects of thawing temperature on the physicochemical properties of pre-rigor frozen chicken breast and leg muscles. Meat Science 71 (2): 375-382. 